\title{
Tentativa suicida y uso del tiempo libre en adolescentes escolarizados mexicanos
}

\author{
Suicide attempt and leisure in Mexican adolescents
}

\author{
Isabel Valadez-Figueroa \\ Universidad de Guadalajara. México. \\ Ana-María Chávez-Hernández \\ Universidad de Guanajuato, México \\ Vania Vargas-Valadez \\ Universidad de Guadalajara. México \\ Quetzalcóatl Hernández-Cervantes \\ Universidad Iberoamericana Puebla, México. \\ María del Consuelo Ochoa-Orendain \\ Universidad de Guadalajara. México.
}

Recibido (20 de octubre de 2017) Aceptado (25 de enero de 2019)

\begin{abstract}
Resumen
El propósito del estudio fue identificar el uso del tiempo libre y su relación con la tentativa suicida en una muestra mexicana adolescente. Participaron 1290 estudiantes de nivel secundaria, entre 12 a 18 años (36\% varones, $64 \%$ mujeres); el instrumento incluyó reactivos cerrados y formato libre. De las 11 categorías del tiempo libre obtenidas, la de relaciones fuera del ámbito familiar obtuvo el mayor puntaje (96\%) y el menor las actividades escolares $(1,8 \%)$. Se encontraron asociaciones estadísticamente significativas de riesgo suicida en las actividades pasivas, del hogar, relaciones familiares y cuidar el propio cuerpo; se encontraron actividades con efecto protector las relaciones sociales fuera del ámbito familiar y recreativas (artísticas, deportivas). Se presentan reflexiones sobre la producción y utilización creativa del tiempo libre en jóvenes y familias contemporáneas.

Palabras clave: Tentativas suicidas; riesgo suicida; tiempo libre; adolescentes; México
\end{abstract}

Correspondencia: Autor correspondiente: Dra. Ana-María Chávez-Hernández. Universidad de Guanajuato. Campus León, Blvd. Puente Milenio 1001, Fracción del Predio San Carlos, CP. 37670, León, Guanajuato. México. anachavez@ugto.mx, anamachavez@hotmail.com Dra. Isabel Valadez-Figueroa. Universidad de Guadalajara, Sierra Mojada 950, Col. Independencia, CP 44348, Guadalajara, Jalisco, México. dravaladez@yahoo.com.mx

Este artículo de investigación científica se deriva del proyecto denominado: "Comportamiento suicida en el adolescente: acercamiento epidemiológico y social", inscrito en el Departamento de Salud Pública del Centro Universitario de Ciencias de la Salud de la Universidad de Guadalajara, Jalisco. Realizado de julio 2015 a mayo del 2016. 


\begin{abstract}
The aim of the present study was to identify leisure time and its association to suicide attempts in a sample of 1290 Mexican adolescents aged 12-18 (36\% male, 64\% female). The instrument included both, closed and open items. Out of 11 leisure categories, relationships outside their families were the most prevalent at $96 \%$ while school-related activities were the least prevalent (at 1.8\%). Statistically significant differences were found for suicidal behavior and passive activities, home activities, family relationships, and body selfcare. Recreation activities such as sports and arts, and relationships outside their families were found to be protective factors. Concluding considerations about the creative use of leisure for contemporary adolescents and families are included.

Keywords: Suicide attempts, suicide risk; leisure time; adolescents; Mexico.
\end{abstract}

\title{
Resumo
}

O objetivo deste estudo foi identificar a relação entre o uso do tempo livre e a tentativa de suicídio em uma amostra de adolescentes mexicanos. Participaram 1290 estudantes de ensino médio, entre 12 e 18 anos (36\% homens, $64 \%$ mulheres). O instrumento de avaliação incluiu perguntas fechadas e abertas. Das 11 categorias estabelecidas para tempo livre, a que obteve maior pontuação (96\%) foi a de relações fora do âmbito familiar e a de menor pontuação (1.8\%) foi de atividades escolares. Foram encontradas associações estatísticas significativas entre o risco de suicídio e atividades passivas, tais como, atividades de casa, relações familiares e cuidados com o corpo próprio; atividades protetoras foram encontradas em relações sociais fora do âmbito familiar e de recreação (artísticas e esportivas). Apresenta-se reflexões sobre a produção e utilização criativa do tempo livre em jovens e famílias contemporâneas.

Palavras-chave: Tentativas suicidas; risco suicida; tempo livre; adolescentes; México.

\section{Introducción}

De acuerdo con la Organización Mundial de la Salud (OMS, 2016) el suicidio consumado por jóvenes entre los 15 a 29 años, se ha posicionado como la segunda causa de muerte a nivel global; la misma fuente considera que las tasas de suicidio han tenido un aumento aproximado del $60 \%$ en los últimos 50 años, con variaciones entre países, considerando además, que estas cifras no incluyen las tentativas de suicidio, que se calculan hasta 20 veces más frecuentes que los casos de suicidio.

En la República Mexicana, al igual que en muchos otros países, la incidencia de las tentativas suicidas presenta un incremento constante a lo largo de la adolescencia, pero con mayor acentuación en el grupo etario de 16 a 18 años (Instituto Nacional de Estadística e Informática [INEGI], 2016); asimismo, algunos estudios han reportado que frecuentemente las tentativas empiezan a presentarse a la edad de 12 años (Chávez-Hernández et al., 2017; QuintanillaMontoya, Sánchez-Loyo \& Pérez, 2015).

El intento suicida forma parte del continuo que caracteriza el comportamiento suicida, que se manifiesta desde la ideación suicida (aspectos cognitivos) hasta el suicidio consumado (aspectos conductuales); pasando por las amenazas o declaraciones verbales, los gestos y tentativas suicidas, hasta el suicidio consumado (Martínez, Gálvez, Quiroz, Vicencio \& Tomicic, 2014). Cuando la persona sobrevive a una tentativa suicida, puede proporcionar información relevante sobre la dinámica personal, familiar, social del fenómeno suicida, entre otros elementos subyacentes; además resulta fundamental darle la atención requerida para evitar futuros actos auto-lesivos, y fortalecer su recuperación y bienestar integral.

\section{Adolescencia, uso del tiempo libre y del ocio}

De acuerdo a la OMS (1998), la adolescencia es clasificada en: adolescencia temprana (10-13 años de edad), adolescencia media (14-16 años de edad), y adolescencia tardía (17-19 años). Durante la adolescencia se cumplen tareas específicas de desarrollo que finalizan con la adquisición de una identidad personal, social, sexual y reproductiva; estableciendo competencias para la vida que hacen posible su autonomía, de tal suerte que a la adolescencia se le considera como un proceso durante el cual se va a ir conformando la identidad del sujeto, es un periodo de descubrimiento de la personalidad, de aprendizaje y toma de decisiones propias. La base psíquica de lo que será su identidad personal es construida mediante la exploración de diferentes modelos de desarrollo, en espacios múltiples y diferentes 
en donde el tiempo libre es un importante contexto de desarrollo (García-Castro \& Pérez-Sánchez, 2010).

Es necesario diferenciar entre tiempo libre y ocio, pues ambos tienen la función de descanso, diversión y desarrollo personal; el tiempo libre es aquél que no está comprometido por obligaciones cotidianas fundamentales ni por las ocupaciones impuestas para la supervivencia como la satisfacción de necesidades básicas (dormir, comer, higiene) o por las responsabilidades sociales así o del trabajo, y es una propuesta externa que le ofrece al individuo una serie de actividades con el fin de entretenerlo (Munné, 1980); el ocio es algo más que el tiempo libre disponible, pues representa experiencias asociadas con actividades que se realizan para la mejora y satisfacción personal, pues es un fin en sí mismo y está ligado a las actividades recreativas (Caride, 2014). Desde una perspectiva psicosocial, el ocio es un modo de comportarse en el tiempo libre, es la parte de ese tiempo libre empleado en hacer cosas que se disfrutan; en sentido estricto, implica subjetividad y vivencias, autonomía y capacidad de decisión de las personas sobre cómo desean emplear su tiempo libre (Cuenca, 2000).

Para el adolescente el tiempo libre tiene especial sentido y pertenencia, durante este tiempo los jóvenes desarrollan actividades cuyas vivencias les ayudan a constituir su sí mismo; es una de las dimensiones fundamentales para el desarrollo de las identidades sociales y personales, por ello se constituye en uno de los elementos definitorios de su personalidad y en el logro de su autonomía, la formación de un auto-concepto y la integración social, si el adolescente fracasa en esta tarea, será susceptible a algunas tendencias autodestructivas, incluyendo el suicidio (Hermosillo De la Torre, Vacio, Méndez-Sánchez, Palacios \& Sahagún, 2015).

Por ende, el propósito de este trabajo fue identificar el uso del tiempo libre en adolescentes de escuelas públicas del nivel medio básico (secundaria) y su asociación con la tentativa suicida; cabe aclarar ha sido poco reportada la relación entre comportamiento suicida y uso del tiempo libre.

\section{Método}

El presente fue un estudio correlacional y transversal; participaron 1290 estudiantes de secundarias públicas de Guadalajara, Jalisco, México; el tamaño de la muestra se determinó con base a la prevalencia de suicidio en adolescentes reportada para Jalisco del 6,6\% (INEGI, 2015), con un nivel de confianza del $95,0 \%$ y un error estimado de $2,7 \%$. La muestra se dividió proporcionalmente en los municipios que conforman la Zona Metropolitana de Guadalajara, centros educativos, turnos y ciclos escolares, y se seleccionaron los estudiantes en forma aleatoria.
El proyecto fue aprobado por el Comité de Ética del Centro Universitario de Ciencias de la Salud de la Universidad de Guadalajara, Jalisco, México. Se explicó a los jóvenes el objetivo del estudio y contenido del instrumento; para todos los sujetos involucrados se recurrió a la participación voluntaria y al consentimiento libre e informado verbal y el anonimato fue contemplado. De forma paralela, a la población de estudio se le proporcionó un directorio de Instituciones a las cuales podían acudir para obtener apoyo psicológico.

El instrumento utilizado se estructuró en cuatro apartados: 1) características sociodemográficas, 2) tiempo libre: actividades que en general realizan en el tiempo libre, la frecuencia de las mismas, y si existe una razón o motivo para realizar dichas actividades y los lugares a los que acude, 3) uso de las redes sociales, tecnologías de la comunicación (teléfonos celulares, computadora), los tipos de programas de televisión y los juegos de video, 4) antecedentes de tentativa suicida.

Para estimar la fuerza de asociación entre el intento suicida y las actividades que realizan en el tiempo libre se utilizó el estadístico OR (conocido como odds ratio en inglés y razón de momios en español que expresa la posibilidad de ocurrencia usando probabilidades). La fuerza de asociación está dada por el número de veces que el valor es superior a uno; además, se calculó el intervalo de confianza (IC) que expresa los límites que con cierta seguridad contendrán ese verdadero valor.

\section{Resultados}

Los estudiantes participantes pertenecían al grupo etario de 12 a 16 años, y cursaban estudios de secundaria. De los 1290 estudiantes que participaron el $10.2 \%(n=132)$ manifestaron antecedentes de intento suicida, correspondiendo el mayor porcentaje al sexo femenino $(63,9 \%)$, lo cual coincide con lo reportado en otros estudios realizados en diferentes regiones de México (Hermosillo De la Torre, Vacio, Méndez-Sánchez, Palacios \& Sahagún, 2015; Hernández-Cervantes \& Alejandre-García, 2015).

Para estos adolescentes el tiempo libre empieza a partir de que salen de la escuela o aun estando en ella, en los espacios de recesos que el centro educativo proporciona dentro de su rutina académica. En los resultados obtenidos por frecuencia de mención, la primera opción señalada de las actividades que realizan en su tiempo libre por los adolescentes, fue el escuchar música.

El $54 \%$ refirió actividades que giraron en torno a las relaciones con pares, planteado como el simplemente estar o salir con los amigos y en algunos especificando además 
salir o estar con su pareja amorosa; casi una quinta parte mencionó actividades que involucran importantemente la tecnología, (televisión, juegos de video, uso de internet y todas las posibilidades que ofrece la red, así como el uso del celular) entre las más citadas se encuentra bajar música, ver películas, utilizar el correo electrónico, chatear, jugar, mensajería instantánea y Facebook; seguido de las actividades que involucran actividad física, como caminar, pasear, patinar, jugar futbol, nadar (20\%) (ver Tabla 1).

Se observó que tanto los hombres como las mujeres, tienen pautas similares en la utilización de su tiempo libre, exceptuando las actividades dedicadas al hogar como ayudar en la casa, y las relacionadas con el cuidado de su cuerpo, las que mayoritariamente fueron mencionadas por las mujeres.

En cuanto al tiempo y frecuencia que dedican a la realización de estas actividades, con respecto a la música es posible considerarlos como oyentes habituales ya que todo el día y todos los días escuchan música; el estar con los amigos casi diario, en donde buena parte de estas relaciones son llevadas a cabo en la escuela en los espacios de receso académico y a la salida del centro escolar (espacios considerados por ellos como tiempo libre); fuera del centro escolar se mencionó en la casa de amigos 2 veces por semana y en el barrio los fines de semana, estar con el novio o novia los fines de semana, realizar actividades fuera de casa dos veces por semana, salir con la familia los fines de semana, días festivos, vacaciones.

Los participantes comentaron que utilizan diariamente la computadora y uso de redes sociales, incluido el teléfono celular y el ver televisión, aproximadamente de 3 a 4 horas diarias o más, según sea los fines de semana, aunque fue especificado como tiempos irregulares a lo largo del día o como fue citado "casi todo el día" además según lo expresado la mayor parte de las veces estas actividades son llevadas a cabo paralelas a la práctica de otras. Con respecto a lo relacionado con el cuidado de su cuerpo, comentaron que ocupan un promedio de una a dos horas diarias las cuales se extienden si salen de casa, con amigos o novio. Por su parte el tiempo que ocupan en realizar las actividades dedicadas al hogar, como cocinar, limpiar la casa, fue en promedio de dos horas diarias o más según sean fines de semana, días de asueto o vacaciones.

Los motivos más destacados para llevar a cabo las actividades, fueron por el gusto y satisfacción de hacerlas, "porque me gusta estar con la banda" por divertirse, "es a todo dar" por hacer ejercicio, para no aburrirse, para salirse de casa, porque me obligan (en el caso de las visitas a fami-

Tabla 1. Frecuencia de mención del uso del tiempo libre

\begin{tabular}{lcc}
\hline Utilización del tiempo libre & No & $\%$ \\
\hline 1. Salir o estar con los amigos/ salir o estar con el novio o novia & 697 & 54.0 \\
2. Ver televisión, juegos de video, internet, chatear, hablar por teléfono & 279 & 21.6 \\
3. Caminar, pasear, patinar, jugar futbol, nadar & 263 & 20.3 \\
4. Leer, escribir, hacer manualidades, coser, confeccionar ropa, dibujar, pintar, pensar & 204 & 15.8 \\
5. Tocar un instrumento musical, cantar en el coro, bailar, tomar clases de baile, modelar, actuar & 204 & 15.8 \\
6. Ir al cine, a fiestas, al box, al futbol, al billar, pasear por centros comerciales, ir de compras, salir a & 191 & 14.8 \\
jugar con mi perro, gato & 153 & 11.8 \\
7. Salir con la familia, estar con la familia, pelear con mi hermano & 96 & 7.44 \\
8. Cocinar, hacer quehacer, arreglar mi cuarto & 31 & 2.40 \\
9. Dormir, comer, bañarse, asearse, descansar, arreglarse (preparar o arreglar la ropa para el día & 27 & 2.09 \\
siguiente, el peinado, y el uso de afeites, antes de salir de casa) & 23 & 1.78 \\
10. Cuidar niños, ayudar en el negocio familiar & 27 \\
11. Estudiar, hacer la tarea & & 2.8 \\
\hline
\end{tabular}

Nota. No $=1290$

Fuente: Elaboración propia. 
liares y actividades del hogar, como condición para obtener permisos) y porque no hay más que hacer.

Acerca de los lugares que frecuentan, fueron señalados la propia casa y el negocio familiar "todos los días"; la escuela "todos los días", el parque, la casa de amigos, la calle "casi todos los días"; unidades deportivas dos o tres veces por semana; la casa de familiares y lugares religiosos para oficios de la religión, cada ocho días; centros comerciales, cine, fines de semana; restaurantes, balnearios por lo regular días feriados y periodos vacacionales.
Las actividades manifestadas que realizan en su tiempo libre se agruparon en 11 categorías tomando en cuenta la frecuencia de mención y de realización de acuerdo al número horas promedio al día que emplean en tales actividades, lo que no excluye que como se dijo en párrafos anteriores, algunas de ellas son llevadas a cabo en forma simultánea (ver Tabla 2).

\section{Tabla 2.Categorías de agrupación del uso del tiempo libre}

\begin{tabular}{ll}
\hline 1. Relaciones fuera del ámbito familiar & Salir o estar con los amigos o salir / estar con el novio o novia. \\
\hline $\begin{array}{l}\text { Pasivas que no estimulan la creatividad (con } \\
\text { bajo nivel de actividad física y de reflexión, } \\
\text { y para exaltar la emoción) }\end{array}$ & Ver televisión, juegos de video, internet, chatear, hablar por teléfono. \\
\hline $\begin{array}{l}\text { Recreativas (divierten, alegran y dependen } \\
\text { de intereses particulares) }\end{array}$ & $\begin{array}{l}\text { Ir al cine, a fiestas, al box, al futbol, al billar, pasear por centros } \\
\text { comerciales, ir de compras, salir a jugar, jugar con mi perro, gato. }\end{array}$ \\
\hline $\begin{array}{l}\text { Pasivas que estimulan la creatividad (sin } \\
\text { actividad física, pero suponen novedad, } \\
\text { estudio y reflexión) }\end{array}$ & $\begin{array}{l}\text { Leer, escribir, hacer manualidades, coser, confeccionar ropa, dibujar, } \\
\text { pintar, pensar. }\end{array}$ \\
\hline $\begin{array}{l}\text { 2. Físico Deportivas } \\
\text { 3. Artísticas }\end{array}$ & Caminar, pasear, patinar, jugar futbol, nadar. \\
\hline $\begin{array}{l}\text { 4. Relaciones familiares } \\
\text { 5. Actividades del hogar }\end{array}$ & $\begin{array}{l}\text { Tocar un instrumento musical, cantar en el coro, bailar, tomar clases de } \\
\text { baile, modelar, actuar. }\end{array}$ \\
\hline 6. Actividades escolares & Salir con la familia, estar con la familia, pelear con mi hermano. \\
\hline 7. Actividades de trabajo & Cocinar, hacer quehacer, arreglar mi cuarto. \\
\hline 8. Actividades relacionadas a cuidar el & Estudiar, hacer la tarea, ir a la escuela. \\
\hline
\end{tabular}

Nota. $\mathrm{N}=1290$

Fuente: Elaboración propia. 
Análisis de Asociación: tiempo libre y mayor riesgo de tentativas suicidas

Resulta relevante señalar que derivado de lo que reportan los participantes, sobre en que ocupan su tiempo libre, se mencionaron las actividades del trabajo, escolares y del hogar por lo que se decidió incluirlas en el análisis. Por otro lado, al ser referido el escuchar música por la totalidad de la población de estudio, se consideró que esta categoría no entrara en el análisis de asociación.

Al relacionar cada una de las categorías de utilización del tiempo libre con antecedente de intento suicida, se encontró asociación con significancia estadística comportándose como factores que incrementan la probabilidad del riesgo para la ocurrencia de intento suicida, las actividades pasivas que no estimulan la creatividad que mostraron la presencia de un riesgo de casi dos veces más para el comportamiento suicida $(\mathrm{OR}=1.67 ; \mathrm{IC}=1.10-2.53)$; las relaciones sociales familiares $(\mathrm{OR}=2.35$; $\mathrm{IC}=1.46-3.77)$; las actividades del hogar $(\mathrm{OR}=2.54 ; \mathrm{IC}=1.44-4.44)$; $\mathrm{y}$ las actividades relacionadas a cuidar el propio cuerpo $(\mathrm{OR}=$ 3.18 ; $\mathrm{IC}=1.28-7.67)$.

Análisis de Asociación: tiempo libre y efecto protector de tentativas suicidas

Así mismo se observó que algunas de las actividades que realizan en su tiempo libre tienen un efecto protector al reducir la probabilidad de riesgo para la ocurrencia de intento suicida, como lo fueron las relaciones sociales fuera del ámbito familiar $(\mathrm{OR}=0.08 ; \mathrm{IC}=0.04-0.15)$; las actividades recreativas en general $(\mathrm{OR}=0.39 ; \mathrm{IC}=0.18-0.81)$ y dentro de ellas las actividades pasivas que estimulan la creatividad $(\mathrm{OR}=0.50 ; \mathrm{IC}=0.26-0.91)$ las artísticas $(\mathrm{OR}=0.23$; $\mathrm{IC}=0.09-0.55)$ y las actividades fisico deportivas $(\mathrm{OR}=$ 0.47 ; $\mathrm{IC}=0.26-0.84$ ) como se muestra en la tabla 3 .

Resultando sin diferencias estadísticas significativas, las actividades escolares $(\mathrm{OR}=1.65 \mathrm{IC}=0.82-3.26)$ y del trabajo $(\mathrm{OR}=2.03$; $\mathrm{IC}=0.66-5.79)$.

Tabla. 3. Asociación entre Tentativa suicida y ocupación del tiempo libre

\begin{tabular}{|c|c|c|c|c|}
\hline Tipo de actividades que realiza & $\begin{array}{l}\text { Intento } \\
\mathrm{N}=132\end{array}$ & $\begin{array}{l}\text { No Intento } \\
\mathrm{N}=1158\end{array}$ & OR & IC \\
\hline \multicolumn{5}{|c|}{$\begin{array}{l}\text { 1. Relaciones sociales fuera del ámbito familiar: salir o estar } \\
\text { con los amigos }\end{array}$} \\
\hline $\mathrm{Si}$ & 14 & 683 & 0.08 & $0.04-0.15$ \\
\hline No & 18 & 475 & & \\
\hline \multicolumn{5}{|c|}{$\begin{array}{l}\text { 2. Pasivas que No estimulan la creatividad } \\
\mathrm{Si}\end{array}$} \\
\hline \multirow[t]{2}{*}{ No } & 40 & 239 & 1.67 & $1.10-2.53$ \\
\hline & 92 & 919 & & \\
\hline \multicolumn{5}{|l|}{ 3. Recreativas } \\
\hline $\mathrm{Si}$ & 9 & 182 & 0.39 & $0.18-0.81$ \\
\hline No & 123 & 976 & & \\
\hline \multicolumn{5}{|c|}{ 4. Pasivas que estimulan la creatividad } \\
\hline $\mathrm{Si}$ & 12 & 192 & & \\
\hline No & 120 & 966 & 0.50 & $0.26-0.91$ \\
\hline \multicolumn{5}{|l|}{ 5. Deportivas } \\
\hline $\mathrm{Si}$ & 15 & 248 & 0.47 & $0.26-0.84$ \\
\hline No & 117 & 910 & & \\
\hline \multicolumn{5}{|l|}{ 6. Artísticas } \\
\hline Alto & 6 & 198 & 0.23 & $0.09-0.55$ \\
\hline Bajo & 126 & 960 & & \\
\hline
\end{tabular}




\begin{tabular}{|c|c|c|c|c|}
\hline Tipo de actividades que realiza & $\begin{array}{l}\text { Intento } \\
\mathrm{N}=132\end{array}$ & $\begin{array}{l}\text { No Intento } \\
\mathrm{N}=1158\end{array}$ & OR & IC \\
\hline $\begin{array}{l}\text { 7. Relaciones sociales - familiares } \\
\mathrm{Si} \\
\text { No }\end{array}$ & $\begin{array}{l}29 \\
103\end{array}$ & $\begin{array}{l}124 \\
1034\end{array}$ & 2.35 & $1.46-3.77$ \\
\hline $\begin{array}{l}\text { 8. Actividades del hogar } \\
\text { Si } \\
\text { No }\end{array}$ & $\begin{array}{l}20 \\
112 \\
\end{array}$ & $\begin{array}{l}76 \\
1082 \\
\end{array}$ & 2.54 & $1.44-4.44$ \\
\hline $\begin{array}{l}\text { 9. Actividades escolares } \\
\mathrm{Si} \\
\text { No }\end{array}$ & $\begin{array}{l}12 \\
120 \\
\end{array}$ & $\begin{array}{l}66 \\
1092 \\
\end{array}$ & 1.65 & $0.82-3.26$ \\
\hline $\begin{array}{l}\text { 10. Actividades de trabajo } \\
\text { Si } \\
\text { No }\end{array}$ & $\begin{array}{l}5 \\
127 \\
\end{array}$ & $\begin{array}{l}22 \\
1136\end{array}$ & 2.03 & $0.66-5.79$ \\
\hline $\begin{array}{l}\text { 11. Actividades relacionadas a cuidar el propio cuerpo } \\
\mathrm{Si} \\
\text { No }\end{array}$ & $\begin{array}{l}8 \\
124\end{array}$ & $\begin{array}{l}23 \\
1135\end{array}$ & 3.18 & $1.28-7.67$ \\
\hline
\end{tabular}

$\mathrm{N}=1290$

Fuente: Elaboración propia

\section{Discusión}

La ocupación del ocio y el tiempo libre, con quienes y en donde se hacen las actividades puede ser un factor de riesgo o un factor de protección para el intento suicida adolescente.

Resulta relevante indicar que derivado de lo reportado resaltan cuatro tipos de actividades que los adolescentes hacen en su tiempo libre, que se comportan como factores de riesgo al incrementar la probabilidad del riesgo para la ocurrencia de intento suicida en el adolescente a saber:

a) Actividades pasivas que no estimulan la creatividad, incrementan casi dos veces la probabilidad de riesgo suicida en el adolescente.

La televisión continúa siendo en los adolescentes una de las principales ocupaciones de su tiempo libre, junto con el empleo de la computadora y su conexión a internet; la televisión es el primer proveedor y trasmisor de valores, promueve la inmediatez y cercanía de los hechos violentos hasta convertirlos en cotidianos, y a su vez mantiene un modelado pasivo de la violencia como medio de resolver conflictos y adquirir poder; en su encuentro regular tiene lugar un aprendizaje de pautas y conductas, potencialmente capaces de modelar sus deseos, creencias, actitudes y comportamientos, lo que conlleva altas posibilidades de ser incorporadas en sus estilos de vida, reproduciendo el com- portamiento observado que se verá reflejado a través de sus manifestaciones en el comportamiento (Bermejo, 2012). La exposición al suicidio o el hecho de morir como una opción viable en la televisión es asociada de manera significativa con autoagresión deliberada; la actitud favorable hacia el suicidio correlaciona positivamente tanto con la ideación suicida como con la tentativa suicida en los adolescentes (Quintanilla-Montoya, Sánchez-Loyo \& Pérez, 2015).

En una breve síntesis de los programas televisivos referidos por esta población, generalmente describen vivencias de otros jóvenes, algunos estereotipos negativos y se incluyen diversos aspectos del género del terror, con grandes dosis de intriga, secretos, mentiras, engaños, traiciones, amenazas, chantajes, peligros, amoríos y una importante carga sexual, algunas formas de violencia o de muerte como algo que pareciera natural; en dichos programas "el hecho de morir se presenta como una alternativa viable, incluso más fuerte que el hecho de seguir viviendo" (Quintanar, 2007, p.21) situaciones que facilitan en el adolescente distorsiones, confusión y devaluación de valores.

En forma paralela Carbonell, Fúster, Chamarro y Oberst (2012) citando a varios autores señalan que el abuso de internet se ha correlacionado con mayores posibilidades de padecer insomnio, disfunción social, malestar psicológico, síntomas somáticos, ansiedad, depresión y pensamientos negativos que interfieren en situaciones sociales, mayor insatisfacción en las relaciones con la familia. 


\section{b) Las relaciones familiares y las actividades en el hogar incrementan más de tres veces la probabili- dad del riesgo para la ocurrencia de intento suicida.}

La llegada de la adolescencia es un momento del ciclo familiar en el que coinciden dos importantes transiciones evolutivas, una en el hijo y otra en sus padres, lo que forzosamente repercutirá en el clima familiar, que se tornará más inestable. En la mayoría de las familias comenzarán a aparecer situaciones con mayores niveles de hostilidad o conflicto con los padres (Nolle, Gulbas, Kuhlberg, \& Zayas, 2012; Paikoff y Brooks-Gunn, 1991). Algunos estudios indican que esos tienen que ver con asuntos cotidianos como los periodos de descanso durante el día, la forma de vestir, el tiempo dedicado al estudio, en qué invierten el tiempo libre, a dónde se va y con quién van, y a qué hora vuelven a casa, esto último se convierte en uno de los aspectos más problemáticos especialmente para las adolescentes mujeres, otro de los asuntos cotidianos va en el sentido del uso de la tecnología (televisión, juegos de video, uso de internet y el uso del celular).

El sexo del adolescente no parece establecer diferencias importantes ni en los niveles globales de conflictividad ni en su evolución, aunque sí el de los padres, ya que son más frecuentes los altercados con las madres (Nolle, Kuhlberg, \& Zayas, 2012; Quilt \& Nava, 2015). Como es el caso de las actividades en el hogar ya que estas son desarrolladas en la casa más frecuentemente por las adolescentes; este trato diferencial suele generar muchas fricciones y conflictos en el hogar, no sólo entre las adolescentes y sus padres sino también entre los propios hermanos y hermanas. Sobre este particular es importante resaltar que debido a las alteraciones del estado del ánimo, la carga emocional que acompaña a estas situaciones es mucho mayor entre la adolescencia inicial y la media (Laursen, Coy \& Collins, 1998), lo que frecuentemente lleva a la huida o retirada del adolescente, dejando el conflicto sin resolver; así, las interacciones familiares desempeñan un papel crucial en el desarrollo del comportamiento suicida particularmente en intentos suicidas.

\section{c. Actividades relacionadas a cuidar el propio cuerpo "el arreglarse" mostraron que incrementan más de tres veces la probabilidad del riesgo para la ocurrencia de intento suicida en el adolescente.}

En esta área es en donde empieza a hacerse evidente el inicio de la adolescencia, puesto que se interesan por otro tipo de ropa, arreglo del cabello e incluso hábitos de higiene. Dada la flojera y apatía, cualquier actividad relacionada con el aseo o cuidado del cuerpo se convierte en una batalla cotidiana, particularmente en los hombres a los que la cul- tura les permite más flexibilidad. Hay que hacer notar que el adolescente atraviesa desequilibrios e inestabilidades que implican transformaciones, una de estas trasformaciones está referida al esquema corporal; en la identificación y conocimiento del propio cuerpo evalúan constantemente con mayor preocupación e inseguridad sus atributos físicos, lo que puede ocasionar descontento de su apariencia personal y un rechazo de sí mismos, particularmente si no cumplen con todas y cada una de las pautas de belleza estéticas y físicas que la moda en vigencia impone por lo que intenta adecuar su esquema a dichas pautas. Para el adolescente su aspecto físico cobra importancia particularmente en esta etapa, puesto que es una dimensión importante de la autoestima y del reconocimiento intersubjetivo por la vía del estilo y la apariencia.

Por su parte de acuerdo a los resultados obtenidos es de destacar las actividades que mostraron comportarse como un factor protección para el intento suicida al disminuir la probabilidad de ocurrencia tales como las relaciones fuera del ámbito familiar, las actividades recreativas, las artísticas y deportivas, o como el escuchar música que diversos estudios han demostrado que actúa como un medio para exteriorizar sus emociones.

\section{A) EL ESCUCHAR MÚSICA}

La música está muy presente en la vida del adolescente y ocupa gran parte de su tiempo de ocio, el escuchar música representa una actividad significativa ya que se asocia a la regulación emocional tanto que actúa como un medio para exteriorizar emociones, como acompañante de las situaciones vitales cotidianas (Ruíz, 2015) y como refuerzo para identificarse con su grupo de pares.

\section{B) LAS RELACIONES FUERA DEL ÁMBITO FAMILIAR EN GENERAL}

Hay que considerar que las relaciones familiares entre el adolescente y su familia se caracterizan por una delicada interacción de procesos paralelos de unión y separación emocional; la transición a la vida adulta implica que los adolescentes dediquen menos tiempo a la familia y más a sus pares, reemplazando las figuras primarias de contacto, el tiempo que pasan con sus amigos se incrementa, al igual que el número y tipo de actividades que comparten con ellos, la relación se va haciendo más intensa y frecuente. Para la mayoría de los y las adolescentes el tiempo libre significa, sobre todo, tiempo para estar con los amigos, de ahí la preferencia por compartir su tiempo libre con el grupo de amigos; la pertenencia a un grupo de amigos y su identificación con ellos es fundamental, hasta tal punto que el ocio se basa más en el hecho de estar con este grupo de amigos que en las actividades que realicen, por lo que gran parte de la vida social de los adolescentes transcurre en el 
ámbito de los grupos, invariablemente en el espacio del tiempo libre. El adolescente valora más el sentirse perteneciente y aceptado en un grupo de pares, que el pasar o estar momentos con sus familiares.

Las actividades pasivas que estimulan la creatividad, las recreativas, así como las artísticas y deportivas, pueden ser entendidas como experiencias de ocio saludable puesto que aportan beneficios concretos; son actividades que se realizan en grupo de pares regularmente y propician la participación protagónica de los y las adolescentes, situación que les genera sentimientos de valía, capacidad, éxito y reconocimiento social, y a su vez incrementan los niveles de autovaloración y de autoestima, constituyen un espacio donde los adolescentes expresan y reconocen sus inquietudes, pensamientos, emociones y sentimientos, además de ejercitar habilidades como la creatividad, la imaginación, el lenguaje, la concentración, el autoconocimiento, memoria e interés por los demás. En cuanto a las actividades deportivas se conoce que la energía que se produce a través del ejercicio físico controla la ansiedad y reduce los niveles de estrés y depresión, mejorando la autoestima y el estado de ánimo (Jiménez, Martínez, Miró y Sánchez, 2008; Arruza, Arribas, Gil De Montes, Irazusta, Romero y Cecchini, 2008).

Todas estas actividades conllevan al adolescente a conocer más gente e interactuar con personas que comparten los mismos intereses, es un proceso de socialización que por intermediación de ellas los adolescentes establecen relación consigo mismos, con su comunidad, con la institución, con los entornos sociales y culturales propios.

\section{Conclusiones}

En este período del desarrollo los jóvenes están en proceso de definir su identidad y su proyecto vital, existe por lo tanto el riesgo de no lograr cumplir exitosamente esta tarea central del desarrollo normal. Durante la adolescencia el uso del tiempo libre puede considerarse con un doble componente, por un lado porque representa un contexto y entorno ideal para la formación humana, a través de dos decisivas variantes, la personal que conduce hacia la conciencia, la responsabilidad y la autodeterminación, y la social que conduce hacia la convivencia y la solidaridad. Y por otra parte representa para el adolescente una situación y oportunidad imprescindible para actuar libremente y un recurso necesario para que se sienta libre y con ello se recupere de las tensiones y frustraciones inherentes a su etapa evolutiva, favoreciendo su equilibrio mental (Castillo-Ceballos, 2005).

Por lo tanto, es de gran importancia considerar los ámbitos familiares, escolares, y de ocio cuya respuesta, siguiendo a Feduchi $(2011$, p.3) tiene una doble función fundamental: "contener las ansiedades expresadas muchas veces en forma de proyecciones, y proponer actividades que el adolescente pueda acoger, junto con otros mecanismos, a través de la identificación introyectiva". Por ello las alternativas de producción, de creación y utilización creativa del tiempo libre es una realidad ineludible para nuestras escuelas, familias y del mundo moderno.

La mayoría de los adolescentes presentan diversos factores que aumentan individualmente su vulnerabilidad a la aflicción emocional y al sufrimiento; elementos que de forma acumulada, pueden dar lugar a comportamientos suicidas. Las actividades que realizan en su tiempo libre, con quienes y donde las hacen, pueden comportarse como una factor de riesgo o un factor de protección para el intento suicida adolescente.

\section{Referencias}

Arruza, J. A., Arribas, S., Gil De Montes, L., Irazusta, S., Romero, S. \& Cecchini, J. A. (2008). Repercusiones de la duración de la Actividad Físico-deportiva sobre el bienestar psicológico. Revista Internacional de Medicina y Ciencias de la Actividad Física y el Deporte, 8, 171-183. Recuperado de http://cdeporte.rediris.es/revista/revista30/ artrepercusiones $83 . \mathrm{htm}$

Bermejo, J. (2012). Los personajes y las series de ficción en la vida de los y las jóvenes. Revista de Estudios de Juventud, 31-49. Recuperado de http://www.injuve.es/sites/default/files/2012/45/publicaciones/ Revista96_2.pdf

Carbonell, X., Fúster, H., Chamarro, A. \& Oberst, U. (2012). Adicción a internet y móvil: una revisión de estudios empíricos españoles. Papeles del Psicólogo, 33, 82-89. Recuperado de http://www. papelesdelpsicologo.es/pdf/2096.pdf

Caride, J. A. (2014). Del ocio como educación social a la pedagogía del ocio en el desarrollo humano. EDETANIA, 33-53. ISSN: 0214-8560. Recuperado de https://dialnet.unirioja.es/descarga/articulo/5010250.pdf

Castillo-Ceballos, G. (2005). El adolescente y sus retos. La aventura de hacerse mayor. Madrid: Ediciones Pirámide.

Chávez-Hernández, A. M., Correa-Romero, F., Acosta-Rojas, I., CardosoEspindola, K. V., Padilla-Gallegos, G. \& Valadez-Figueroa, I. (2017). Suicidal Ideation, Depressive Symtomatology and SelfConcept: A comparison between Mexican Institucionalized and Non institucionalized Children. Suicide and Life-Threatening Behavior, early view. doi: 10.1111/sltb. 12340

Cuenca, M. (2000). Dimensiones y manifestaciones actuales del ocio. Bilbao: Universidad de Deusto.

Feduchi, L. (2011). El adolescente ante su futuro. Temas de psicoanálisis, 1-11. Recuperado de http://www.temasdepsicoanalisis.org/wp-content/ uploads/2010/12/el-adolescente-ante-su-futuro.pdf

García-Castro, J. \& Pérez-Sánchez, R. (2010). Tiempo libre en adolescentes escolarizados de dos clases sociales de Costa Rica. Rev. Latinoamericana de Ciencias Sociales, Niñez y Juventud, 8, 439-454. Recuperado de http:// 
biblioteca.clacso.edu.ar/Colombia/alianza-cinde-umz/20131216052335/ art.JuanDiegoG.pdf

Hermosillo De la Torre, E., Vacio Muro, M., Méndez-Sánchez, C., Palacios Salas, P. \& Sahagún Padilla M. (2015). Sintomatología depresiva, desesperanza y recursos psicológicos: una relación con la tentativa de suicidio en una muestra de adolescentes mexicanos. Acta Universitaria, 25, 52-56. doi: 10.151747/au.2015.900

Hernández-Cervantes, Q. \& Alejandre-García, J. (2015). Relación entre riesgo suicida y uso de tabaco o alcohol en una muestra de universitarios. Acta Universitaria, 25, 47-51. doi:10.15174/au.2015.899

Instituto Nacional de Estadística y Geografía [INEGI]. (2015). Estadísticas de mortalidad. Recuperado de http://www.inegi.org.mx

Instituto Nacional de Estadística y Geografía [INEGI]. (2016). Estadísticas de mortalidad. Recuperado de http://www.inegi.org.mx

Jiménez, M., Martínez, P., Miró, E. \& Sánchez, A. (2008). Bienestar psicológico y hábitos saludables: ¿están asociados a la práctica dle ejercicio físico? Int J Clin Health Psychol, 8, 185-202. Recuperado de http://www.redalyc.org/pdf/337/33780113.pdf

Laursen, B., Coy, K. C. \& Collins, W. A. (1998). Reconsidering changes in parent-child conflict across adolescence: A meta-analysis. Child Development, 69, 817-832. doi: 10.1111/j.1467-8624.1998.tb06245.x

Martínez, C., Gálvez, C., Quiroz, C., Vicencio, B. \& Tomicic, A. (2014). He Decidido Vivir: Factores que disuaden a una persona de cometer suicidio. Revista Argentina de Clínica Psicológica, XIII, 267-280. Recuperado de https://dialnet.unirioja.es/servlet/articulo?codigo=5427130

Munné, F. (1980). Psicosociología del tiempo libre. Un enfoque crítico. México, D.F.: Trillas.

Nolle, A.P., Gulbas, L., Kuhlberg, J. \& Zayas, L. (2012). Sacrifice for the sake of the family: Expressions of familism by Latin teens in the context of suicide. American Journal of Orthopsychiatry, 82, 319-327. doi:10.111/j.1939-0025.2012.01166.x.

Organización Mundial de la Salud [OMS]. (1998). Manual de monitoreo y evaluación de programas de salud sexual y reproductiva de adolescente y jóvenes. ( $\mathrm{N}^{\circ} 3$, Tomo 1). Caracas, Venezuela: Publicaciones Ministerio de Sanidad y Asistencia Social (MSAS).

Organización Mundial de la Salud [OMS]. (2016). Global Health Estimates 2014 Summary Tables: Deaths by Cause, Age and Sex, 2000-2012. Recovered of http://www.who.int/healthinfo/global_burden_disease/ estimates/en/index1.html

Paikoff, R. L. \& Brooks-Gunn, J. (1991). Do parent-child relationships change during puberty? Psychological Bulletin, 110, 47-66. doi: 10.1037//0033-2909.110.1.47

Quilt Meléndez, M. \& Nava Ernult, A. (2015). Ideación Suicida y su relación con el funcionamiento familiar y diferenciación familiar en jóvenes universitarios tlaxcaltecas. Acta Universitaria, 25, 70-74. doi: 10.15174/au.2015.871.
Quintanilla-Montoya, R., Sánchez-Loyo, M. \& Pérez Lúa, I. (2015). Conceptos de muerte y suicidio en una muestra de menores mexicanos de 5 a 14 años de edad. Acta Universitaria, 25, 24-28. doi: 10.15174/ au.2015,887.

Quintanar, F. (2007). Comportamiento suicida, perfil psicológico y posibilidades de tratamiento. México: Pax México.

Ruiz, A. (2015). El papel de la música en la construcción de una identidad durante la adolescencia ¿Dime qué escuchas y te diré quién eres? SINERIS, Revista de musicología, 1-42. ISSN 2254-3643. Recuperado de http://www.sineris.es/adolescentes.pdf 